\title{
Analysis of UAV Kinematic Constraints for Rigid Formation Flying
}

\author{
Vinay Reddy Challa* and Ashwini Ratnoo ${ }^{\dagger}$ \\ Indian Institute of Science, Bangalore, 560 012, India
}

\begin{abstract}
The problem of multi-UAV rigid formation flying manoeuvres is considered. In a leaderfollower frame work, an analysis is carried out for flyable operating region considering bank angle, stalling speed and maximum speed constraints. Speed and bank angle requirements for a wingman are derived with respect to prospective leader manoeuvre and the formation geometry. Further, the work uses these relations and obtains a closed form operating region for the leader imposed by all the wingmen constraints. The flyable region is presented in speed - turn radius space. Simulations are carried out validating the analytic findings. The work presents a simple and closed-form design methodology for feasible UAV formation manoeuvres.
\end{abstract}

\section{Introduction}

UAVs are used for various applications due to rising level of autonomy. One such application, formation flying, involves two or more UAVs to fly together in a particular geometry to accomplish a mission or a task. Formation flying finds applications in areas like reconnaissance missions, military applications, search missions in wider formations, fuel saving in closer formation and target triangulation using vision based approaches. Common approaches for formation flying guidance include leader-follower approach, ${ }^{1-4}$ variable structure approach, ${ }^{5-7}$ etc. In leader-follower approach, one UAV is designated as the leader and the task of the other UAVs is to follow the leader while maintaining a particular distance and bearing with respect to the leader. Maintaining rigidity of formation is critical for collision avoidance and safe operations. Rigid formation also makes missions like target triangulating and surveillance easier to implement.

Pachter et al. ${ }^{2}$ proposed a formation-hold autopilot using leader-follower approach. Formation generation guidance laws using line-of-sight guidance principles were proposed by Tahk et al. ${ }^{1}$ Another leader-follower approach using vector fields was proposed by Kwon and Chwa. ${ }^{12}$ Variable structure method for mobile robots was discussed by Tan and Lewis. ${ }^{9}$ Flexible and dynamic variable structure methods for formation of UAVs were introduced by Low ${ }^{10}$ and Low and Ng. ${ }^{7}$ Formation maintenance with obstacle avoidance, collision avoidance, formation manoeuvrability, etc. were discussed by Moscovitz and DeClaris. ${ }^{8}$ Formation manoeuvrability means the ability of formation to manoeuvre while maintaining rigidity. The trajectory of a fixed wing UAV level flight has two operational constraints or states namely, airspeed and bank angle. These states have saturation limits hereinafter referred to as kinematic constraints. As a formation manoeuvres, rigidity imposes different speed and turn rate requirements for the vehicles depending on the relative geometry. A closed-form relationship for the manoeuvrability of the formation can be defined in the leader-follower framework.

The motivation of this work is to find the operating region for the leader which, for a given formation manoeuvre, keeps all follower UAVs within their kinematic constraints. As the main contributions of this work kinematics of two-UAV formation is analysed for a rigid formation manoeuvre. The kinematic constraints and formation kinematics are analysed to define an operating region for a two UAV rigid formation. These relations are further analysed to obtain a closed-form operating region for the leader imposed by the

\footnotetext{
*Research Student, Autonomous Vehicles Laboratory, Department of Aerospace Engineering, Indian Institute of Science, Bangalore, 560 012, India, vinayreddych89@gmail.com

${ }^{\dagger}$ Assitant Professor, Autonomous Vehicles Laboratory, Department of Aerospace Engineering, Indian Institute of Science, Bangalore, 560 012, India, ratnoo@aero.iisc.ernet.in
} 
all wingmen constraints. The flyable region is presented in turn radius-speed space. Simulations for different operating points are presented to validate the analytic findings.

Reminder of the paper is organised as follows: In Section II, kinematic constraints and formation kinematics have been analysed. Section III presents derivation of curves defining operating region for two UAVs in rigid formation. The results obtained in Section III are extended to multiple UAV formation manoeuvre in Section IV. Simulation studies are presented for operating points within and outside the operating region in Section V. Concluding remarks are given in Section VI.

\section{Kinematic constraints and two-UAV formation}

\section{A. Kinematic constraints}

Fixed wing UAVs are nonholonomic systems, and have limitations on their turn rate and speed. For a level flight, the weight of the UAV is balanced by vertical component of lift force and the horizontal component of the lift force $L$ provides the necessary centripetal force. A schematic of forces is shown in figure 1 . The UAV turns with a radius $R$ by maintaining a roll angle $\phi$. From figure 1, it can be deduced that,

$$
\begin{aligned}
& L \cos \phi=W=m g \\
& L \sin \phi=m a_{\text {lat }}
\end{aligned}
$$

Equation (2) shows that a larger roll angle $\phi$ produces more centripetal acceleration $a_{\text {lat }}$. This leads to a

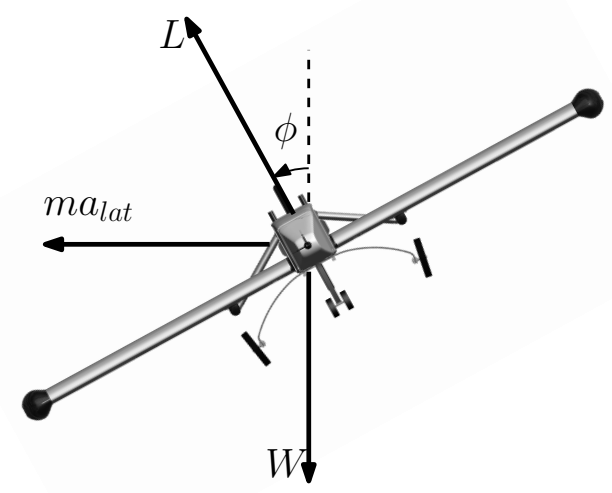

Figure 1. UAV level turn.

requirement of a greater lift force, as governed by Eq. (1), for a level flight. Consequently, if $L_{\max }$ is the maximum lift force generated by the UAV, the roll angle limitation $\phi_{\text {lim }}$ can be deduced by using Eq. (1) as,

$$
\phi_{\text {lim }}=\cos ^{-1}\left(\frac{m g}{L_{\max }}\right)
$$

Typically $\phi_{\max }$, the maximum roll angle of UAV, is chosen as $\phi_{\max }<\phi_{\text {lim }}$.

Lift force is dependent on the airspeed of UAV, and there exists minimum airspeed called stall speed $V_{\text {stall }}$ at which aircraft starts stalling, it is unable to generate the required lift. The minimum speed $V_{\text {min }}$, of the UAV is chosen such that UAV can maintain maximum roll angle $\phi_{\max }$ without stalling. Also, a limited thrust limits the maximum airspeed of UAV. $V_{\max }$ is the maximum airspeed of UAV, and is achieved at maximum throttle. Parameters $V_{\max }, V_{\min }$ and $\phi_{\max }$ define the three limiting factors of fixed wing UAV manoeuvrability. 
Other parameters can be derived from these three parameters. Dividing Eq. (2) by Eq. (1). we get,

$$
\begin{aligned}
\tan \phi & =\frac{a_{\text {lat }}}{g} \\
\Rightarrow a_{\text {lat }} & =g \tan \phi
\end{aligned}
$$

where, $a_{l a t}$ is the centripetal acceleration which also can be represented as

$$
\begin{array}{r}
\frac{V^{2}}{R}=a_{\text {lat }}=g \tan \phi \\
\Rightarrow R=\frac{V^{2}}{g \tan \phi}
\end{array}
$$

Equation (7) shows that, a higher bank angle is required for tighter turns. As bank angle $(\phi)$ is limited by $\phi_{\max }$, Eq. (7) can be arranged to deduce as,

$$
R \geq \frac{V^{2}}{g \tan \phi_{\max }}
$$

which can be used to determine a curve $\mathcal{R}^{\phi_{\max }}$ in speed-bank angle space as,

$$
\mathcal{R}^{\phi_{\max }} \equiv V^{2}-g R \tan \phi_{\max } \leq 0
$$

Curve $\mathcal{R}^{\phi_{\max }}$ represents the bank angle limitation for an individual UAV. In a region bounded by $\mathcal{R}^{\phi_{\text {max }}}$ the UAV can manoeuvre without saturating in bank angle. Based on bank angle limitation curve and speed constraints, the operating region for an individual UAV is shown in figure 2. Turn rate $\dot{\gamma}$ is given by,

$$
\dot{\gamma}=\frac{V}{R}
$$

Using Eq. (7) in Eq. (9) leads to

$$
\Rightarrow \dot{\gamma}=\frac{g \tan \phi}{V}
$$

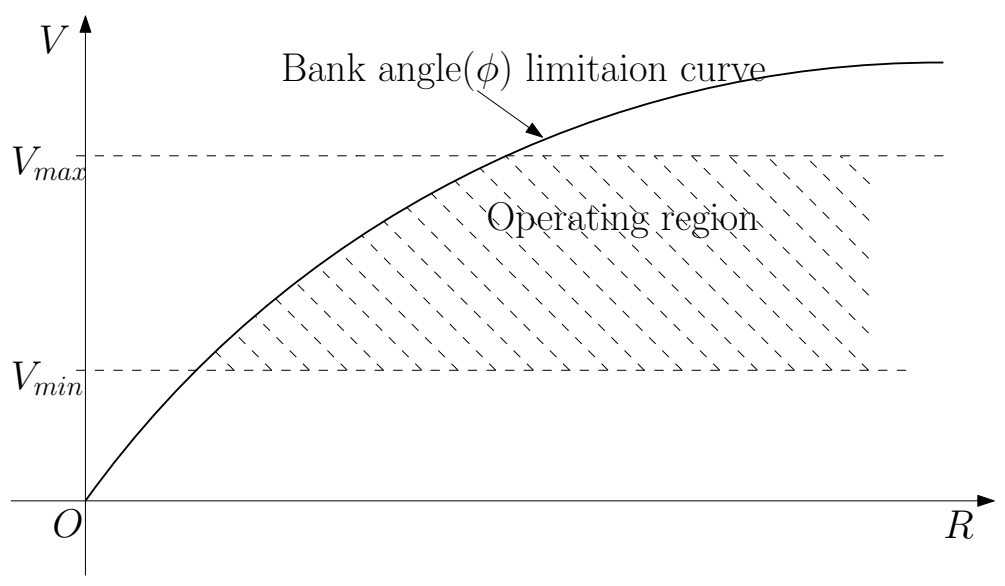

Figure 2. Operating region for a single UAV. 


\section{B. Formation kinematics}

To derive kinematic relations between UAVs in formation, consider two UAVs (leader-follower) as shown in figure 3 manoeuvring in a rigid formation. For a rigid formation, the distance $\rho_{c}$ between UAVs and relative orientation $\theta_{c}$ with respect to leader's heading should be constant, that is,

$$
\begin{aligned}
& \dot{\rho}_{c}=0 \\
& \dot{\theta_{c}}=0
\end{aligned}
$$

The kinematic equations for the formation can be written as,

$$
\begin{aligned}
\dot{\rho}_{c} & =V_{l} \cos \theta_{c}-V_{w} \cos \theta \\
\rho_{c}\left(\dot{\theta}_{c}+\dot{\gamma}_{l}\right) & =-V_{l} \sin \theta_{c}+V_{w} \sin \theta
\end{aligned}
$$

Here, $V_{l}$ and $\gamma_{l}$ are the speed and heading of the leader, respectively and similarly, $V_{w}$ and $\gamma_{w}$ are the speed and heading of wingman, respectively. $R_{l}$ represents the instantaneous turn radius of the leader.

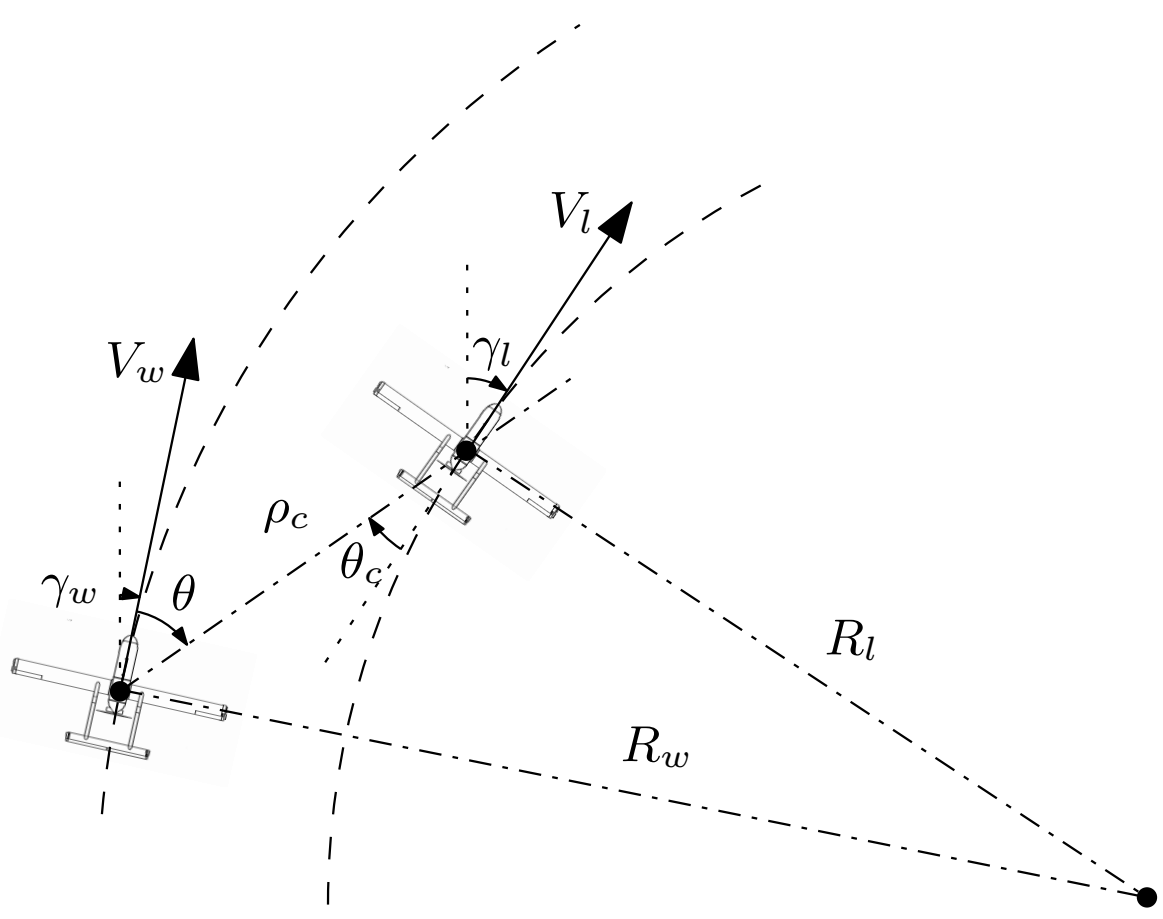

Figure 3. Formation geometry.

Where, $\dot{\gamma}_{l}=\frac{V_{l}}{R_{l}}$ and $\theta=\gamma_{l}+\theta_{c}-\gamma_{w}$. Imposing Eq. (11) on Eq. (12) and Eq. (13) it can be deduced that for a rigid formation,

$$
\begin{aligned}
& V_{w} \cos \left(\gamma_{l}+\theta_{c}-\gamma_{w}\right)=V_{l} \cos \theta_{c} \\
& V_{w} \sin \left(\gamma_{l}+\theta_{c}-\gamma_{w}\right)=\rho_{c} \frac{V_{l}}{R_{l}}+V_{l} \sin \theta_{c}
\end{aligned}
$$

Equations (14) and (15) can be solved for $\gamma_{w}$ and $V_{w}$ as,

$$
\begin{aligned}
& \gamma_{w}=\gamma_{l}+\theta_{c}-\tan ^{-1}\left(\frac{\sin \theta_{c}+\frac{\rho_{c}}{R_{l}}}{\cos \theta_{c}}\right) \\
& V_{w}=V_{l} \sqrt{1+\frac{\rho_{c}^{2}}{R_{l}^{2}}+2 \frac{\rho_{c}}{R_{l}} \sin \theta_{c}}
\end{aligned}
$$


Equations (16) and (17), govern the required heading and speed of the follower to maintain the desired formation. Differentiating Eq. (16) with respect to time results in,

$$
\dot{\gamma_{w}}=\dot{\gamma}_{l}
$$

From Eq. (18) it is evident that for UAVs in formation, the turn rates should be the same. This can also be understood as the time taken for one complete turn should be same for both the vehicles. In the following section, two UAV formation is considered and the effect of kinematic constraints obtained in this section on rigidity of the formation is analysed.

\section{Operating Region for two UAVs in rigid formation}

The objective here is to find an operating region for the leader which guarantees a within flyable limit operation for all the wingmen. To establish operating region for a rigid formation, the formations kinematics derived in previous section are analysed along with the UAV level turn limits. Consider two UAVs flying in rigid formation as shown in figure 3. Let $R_{w}$ and $R_{l}$ be instantaneous turn radius of wingman and leader, respectively. The relation between the velocities and heading of wingman and leader are given in Eq. (16) and Eq. (17). From Eq. (18) it can be deduced that,

$$
\begin{aligned}
\frac{V_{w}}{R_{w}} & =\frac{V_{l}}{R_{l}} \\
\Rightarrow R_{w} & =\frac{V_{w}}{V_{l}} R_{l}
\end{aligned}
$$

Using Eq. (19) in Eq. (17) leads to,

$$
R_{w}=R_{l} \sqrt{1+\frac{\rho_{c}^{2}}{R_{l}^{2}}+2 \frac{\rho_{c}}{R_{l}} \sin \theta_{c}}
$$

Rewriting Eq. (20) as,

$$
R_{w}=k R_{l}
$$

where

$$
k=\sqrt{1+\frac{\rho_{c}^{2}}{R_{l}^{2}}+2 \frac{\rho_{c}}{R_{l}} \sin \theta_{c}}
$$

Similarly, using Eq. (10) in Eq. (18) results in,

$$
\tan \phi_{w}=k \tan \phi_{l}
$$

Note that for $k>1$, using Eq. (21) $R_{w}>R_{l}$, implies that wingman's instantaneous turn radius is larger than that of the leader and it is referred to as outer UAV. Similarly If $k \leq 1$, then $R_{w} \leq R_{l}$ that is wingman instantaneous turn radius is smaller than or equal to that of the leader and therefore called inner UAV. Analysis for the operating region with outer and inner UAVs is presented in the following subsections.

\section{A. Operation region constraints due to an outer wingman $(k>1)$}

In this case, wingman is an outer UAV with $k>1$. From Eq. (23) for $k>1, \phi_{w}>\phi_{l}$, the wingman requires a higher bank angle. Let $V_{l}^{*}, R_{l}^{*}$ and $\phi_{l}^{*}$ be the leader's states at which the wingman bank angle reaches its maximum value. Using Eq. (23) it can be deduced that,

$$
\tan \phi_{l}^{*}=\frac{\tan \phi_{\max }}{k}
$$

Using Eq. (7) and Eq. (24) implies

$$
\begin{aligned}
V_{l}^{* 2} k & =g R_{l}^{*} \tan \phi_{\text {max }} \\
\Rightarrow V_{l}^{* 2} \sqrt{1+\frac{\rho_{c}^{2}}{R_{l}^{* 2}}+2 \frac{\rho_{c}}{R_{l}^{*}} \sin \theta_{c}} & =g R_{l}^{*} \tan \phi_{\text {max }}
\end{aligned}
$$


Rearranging Eq. (26) leads to,

$$
V_{l}^{* 4} R_{l}^{* 2}+2 \rho_{c} V_{l}^{* 4} R_{l} \sin \theta_{c}^{*}+V_{l}^{* 4} \rho_{c}^{2}-g^{2} \tan ^{2}\left(\phi_{\max }\right) R_{l}^{* 4}=0
$$

This equation represents a fourth degree polynomial curve in $\left(R_{l}, V_{l}\right)$ space and is called bank angle saturation curve. The corresponding region $\mathcal{R}_{W}^{\phi_{\max }}$ in $\left(R_{l}, V_{l}\right)$ space, where wingman can maintain formation without reaching banking saturation $\phi_{w} \leq \phi_{\max }$ is given by,

$$
\mathcal{R}_{W}^{\phi_{\max }} \equiv V_{l}^{* 4} R_{l}^{* 2}+2 \rho_{c} V_{l}^{* 4} R_{l} \sin \theta_{c}^{*}+V_{l}^{* 4} \rho_{c}^{2}-g^{2} \tan ^{2}\left(\phi_{\max }\right) R_{l}^{* 4} \leq 0
$$

This region represents the operating region for the leader UAV such that the follower doesn't saturate in bank angle. This region should be subset of $\mathcal{R}^{\phi_{\max }}$ as any UAV is bounded by Eq. (8). This is proven in the following lemma.

Lemma III.1. For rigid $U A V$ formation, if there exists at least one follower $W$ with $k>1$, then $\mathcal{R}_{W}^{\phi_{\max }} \subset$ $\mathcal{R}^{\phi_{\max }}$, where $\mathcal{R}^{\phi_{\max }}$ is bank angle saturation region of the leader and $\mathcal{R}_{W}^{\phi_{\max }}$ is the bank angle limit imposed on the leader by an outer $U A V$.

Proof. Consider an outer UAV in rigid formation with leader. Let $\left(R_{l}^{*}, V_{l_{W}}^{*}\right)$ be the operating point of the leader where follower saturates in bank angle and $\left(R_{l}^{*}, V_{l}^{*}\right)$ be the operating point where leader itself saturates in bank angle. From Eq. (25) we can write,

$$
\begin{gathered}
V_{l_{W}}^{* 2}=\frac{g R_{l}^{*} \tan \phi_{\max }}{k} \\
k>1 \\
\frac{g R_{l}^{*} \tan \phi_{\max }}{k}<g R_{l}^{*} \tan \phi_{\max } \\
V_{l_{W}}^{* 2}<V_{l}^{* 2} \\
V_{l_{W}}^{*}<V_{l}^{*}
\end{gathered}
$$

Equation (50) shows that every saturation point $\left(R_{l}^{*}, V_{l_{W}}^{*}\right)$ lies within the region $\left(R_{l}^{*}, V_{l}^{*}\right)$. Therefore,

$$
\mathcal{R}_{W}^{\phi_{\max }} \subset \mathcal{R}^{\phi_{\max }}
$$

Lemma III.1 shows that inner wingmen bank angle is within flyable limits for any leader bank angle within its own flyable limits. Next, the speed requirements for such a formation $(k>1)$ are investigated. From Eq. (17) for $k>1$,

$$
V_{w}>V_{l}
$$

Consider the leader's states as $V_{l}^{*}, R_{l}^{*}$ and $\phi_{l}^{*}$ which requires the wingman to use the maximum speed, that is, when $V_{w}=V_{\max }$. Rewriting Eq. (17) leads to

$$
\begin{array}{r}
V_{\max }=V_{l}^{*} \sqrt{1+\frac{\rho_{c}^{2}}{R_{l}^{* 2}}+2 \frac{\rho_{c}}{R_{l}^{*}} \sin \theta_{c}} \\
\Rightarrow R_{l}^{* 2} V_{\text {max }}^{2}-V_{l}^{* 2} R_{l}^{* 2}-V_{l}^{* 2} \rho_{c}^{2}-2 V_{l}^{* 2} \rho_{c} \sin \theta_{c} R_{l}^{*}=0
\end{array}
$$

For $V_{w}<V_{\max }$ a region $\mathcal{R}_{W}^{V_{\max }}$, hereinafter known as maximum airspeed saturation region, can be obtained from Eq. (32) as

$$
\mathcal{R}_{W}^{V_{\max }} \equiv V_{l}^{2} R_{l}^{2}+V_{l}^{2} \rho_{c}^{2}+2 V_{l}^{2} \rho_{c} R_{l}-R_{l}^{2} V_{\max }^{2} \leq 0
$$

A leader flying in this region ensures that the follower requires a speed less than the maximum airspeed. In case of two UAV formation an with outer follower, the operating region to ensure both $V_{w} \leq V_{\text {max }}$ and $\phi_{w} \leq \phi_{\max }$ is the obtained intersection of the regions $\mathcal{R}_{W}^{\phi_{\max }}$ and $\mathcal{R}_{W}^{V_{\max }}$. A leader operation within the region $\mathcal{R}^{\text {outer }} \equiv \mathcal{R}_{W}^{\phi_{\max }} \cap \mathcal{R}_{W}^{V_{\max }}$ guarantees rigid formation with an outer wingman $(k>1)$. This region could 


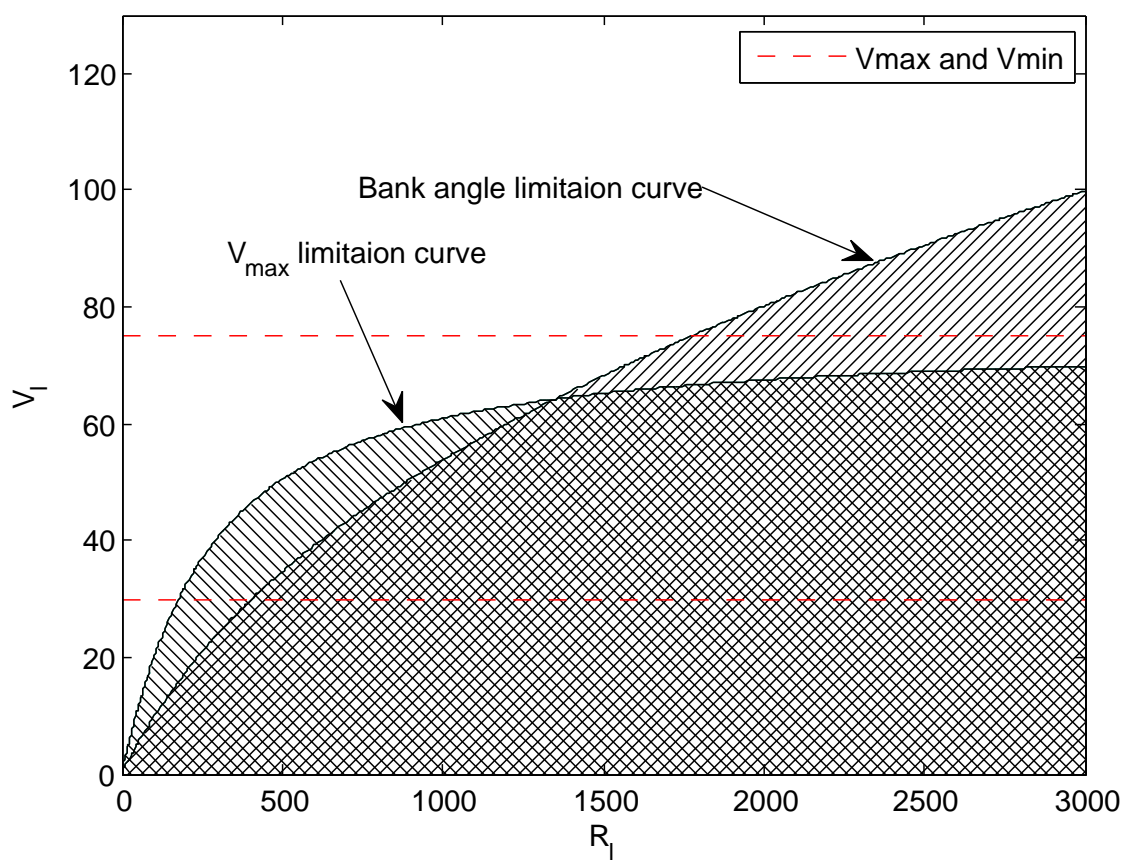

Figure 4. Operating region of leader with an outer UAV $(k>1)$.

be explained as the conditions imposed by outer UAV on the leader to maintain rigidity. The intersection region for an example scenario with $V_{\max }=75 \mathrm{~m} / \mathrm{s}, V_{\min }=30 \mathrm{~m} / \mathrm{s}, \phi_{\max }=20^{\circ}, \rho_{c}=300 \mathrm{~m}$ and $\theta_{c}=45^{\circ}$ is shown in figure 4 . The bank angle limitation curve and $V_{\max }$ limitation curve are also shown. Note that the speed saturation curve is asymptotic to $V_{\max }$ and touches $V_{\max }$ at $R_{l}=\infty$, which is a non-manoeuvring formation.

\section{B. Operating region constraints due to inner $\operatorname{wingman}(k \leq 1)$}

In this case an inner wingman, using Eq. (23) with $k \leq 1$ results in $\phi_{w} \leq \phi_{l}$, the wingman bank angle is less than that of the leader. Hence there is no bank angle limitation imposed by the inner followers on the leader From Eq. (15), the wingman's speed $V_{w}<V_{l}$, for $k \leq 1$. Hence, the inner wingman moves with a slower speed as compared to the leader. Consider the leader speed, turn radius and bank angle as $V_{l}^{*}, R_{l}^{*}$ and $\phi_{l}^{*}$ which correspond to the inner wingman stall speed. Hence, using Eq. (17),

$$
\begin{array}{r}
V_{\min }=V_{l}^{*} \sqrt{1+\frac{\rho_{c}^{2}}{R_{l}^{* 2}}+2 \frac{\rho_{c}}{R_{l}^{*}} \sin \theta_{c}} \\
\Rightarrow R_{l}^{* 2} V_{\text {min }}^{2}-V_{l}^{* 2} R_{l}^{* 2}-V_{l}^{* 2} \rho_{c}^{2}-2 V_{l}^{* 2} \rho_{c} \sin \theta_{c} R_{l}^{*}=0
\end{array}
$$

For $V_{w}<V_{\text {min }}$ a region $\mathcal{R}_{W}^{V_{\text {min }}}$, hereinafter known as minimum airspeed saturation region, can be obtained from Eq. (35) as

$$
\mathcal{R}_{W}^{V_{\text {min }}} \equiv V_{l}^{2} R_{l}^{2}+V_{l}^{2} \rho_{c}^{2}+2 V_{l}^{2} \rho_{c} R_{l}-R_{l}^{2} V_{\text {min }}^{2} \geq 0
$$

As the inner wingman does not impose a bank angle constraint on the leader, $\mathcal{R}^{\phi_{\max }}$ limits the bank angle operating region for the leader. The operating region in airspeed-turn radius space is determined by the intersection of $\mathcal{R}_{W}^{V_{\text {min }}}$ and $\mathcal{R}^{\phi_{\max }}$ regions. Therefore a leader flying within the region $\mathcal{R}^{\text {inner }} \equiv \mathcal{R}^{\phi_{\max }} \cap \mathcal{R}_{W}^{V_{\min }}$ guarantees rigid formation with inner wingman $(k \leq 1)$. The intersection region for the example scenario one 
with $\theta_{c}=-45^{\circ}$ is shown in figure 5 . Note that the $V_{\min }$ saturation curve asymptotically reaches $V_{\min }$ and touches $V_{\min }$ as $R_{l}$ tends to infinity, which corresponds to a non-manoeuvring formation.

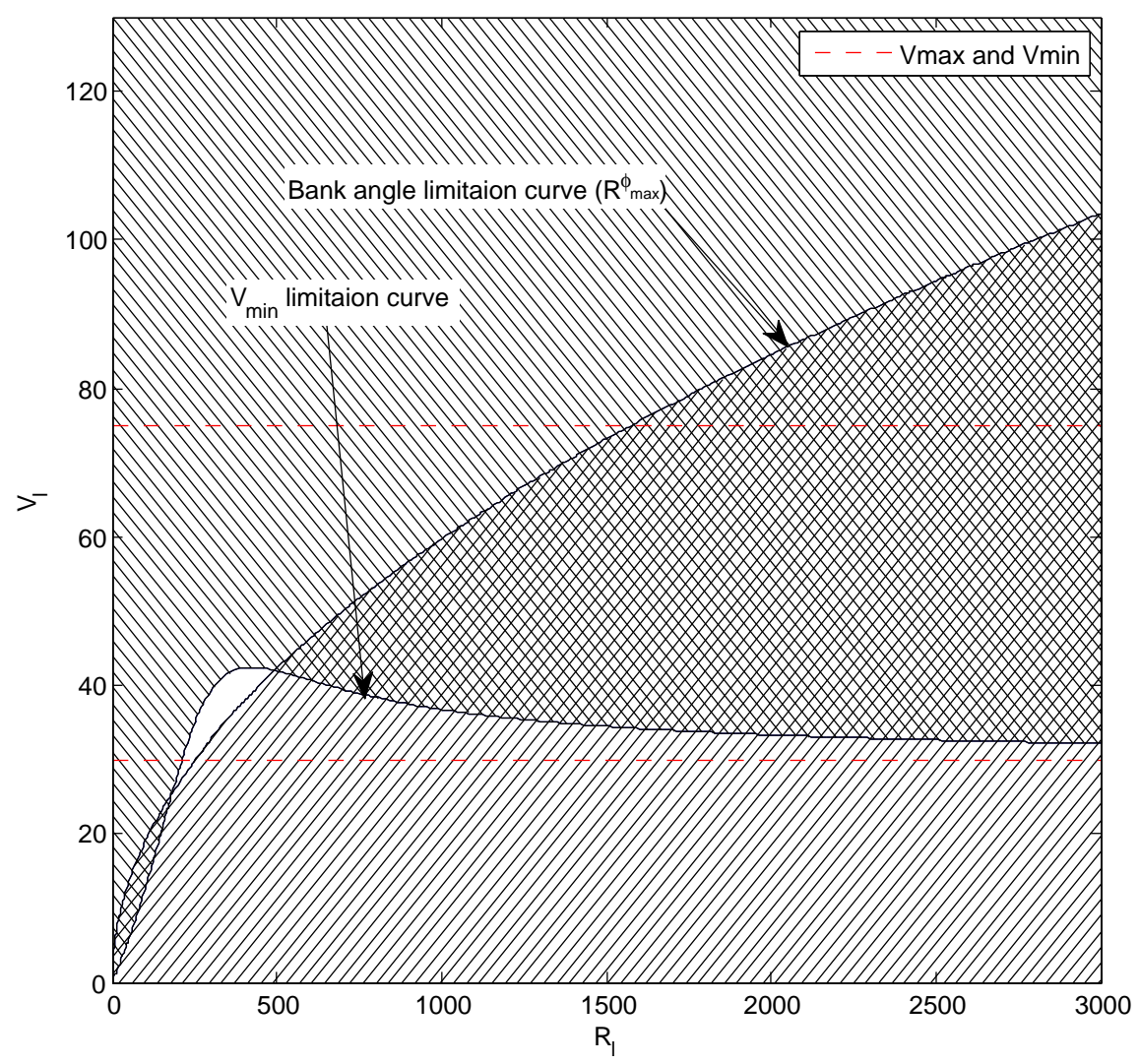

Figure 5. Operating region of leader with an inner UAV $(k \leq 1)$.

\section{Operating region for multiple UAVs}

Based on the individual analysis on inner and outer wingman configurations, this section deduces the leader operating region for multiple $\operatorname{UAVs}(>2)$. For a given formation configuration, the inner most and outer most UAVs are defined as UAVs with largest and smallest instantaneous turn radius, respectively. The outermost UAV has highest $k$ and the innermost UAV has the least $k$. To define the operating region for multiple UAVs, the following lemmas are used.

Lemma IV.1. If $W_{1}, W_{2}$ are two outer followers with $k_{1}, k_{2}$ such that $k_{1}>k_{2}>1$, then $\mathcal{R}_{W_{1}}^{\phi_{\max }} \subset \mathcal{R}_{W_{2}}^{\phi_{\max }}$. Where $\mathcal{R}_{W_{1}}^{\phi_{\max }}$ and $\mathcal{R}_{W_{2}}^{\phi_{\max }}$ are leader bank angle saturation regions corresponding to $W_{1}$ and $W_{2}$, respectively.

Proof. Consider two outer UAVs $W_{1}$ and $W_{2}$ with formation parameters as $\rho_{c 1}, \theta_{c 1}$ and $\rho_{c 2}, \theta_{c 2}$, respectively. Let $\left(R_{l}^{*}, V_{l_{W_{1}}}^{*}\right)$ be the leader operating point where $W_{1}$ saturates in bank angle and $\left(R_{l}^{*}, V_{l_{W_{2}}}^{*}\right)$ be the operating point where $W_{2}$ saturates in bank angle. Using Eq. (25) for the two operating points,

$$
\begin{aligned}
V_{l_{W_{1}}}^{* 2} & =\frac{g R_{l}^{*} \tan \phi_{\max }}{k_{1}} \\
V_{l_{W_{2}}}^{* 2} & =\frac{g R_{l}^{*} \tan \phi_{\max }}{k_{2}}
\end{aligned}
$$


Since $k_{1}>k_{2}$, it implies

$$
\frac{g R_{l}^{*} \tan \phi_{\max }}{k_{1}}<\frac{g R_{l}^{*} \tan \phi_{\max }}{k_{2}}
$$

Using Eq. (37) and Eq. (38) in Eq. (39),

$$
\begin{aligned}
V_{l_{W_{1}}}^{*}<V_{l_{W_{2}}}^{*} \\
\Rightarrow \mathcal{R}_{W_{1}}^{\phi_{\max }} \subset \mathcal{R}_{W_{2}}^{\phi_{\max }}
\end{aligned}
$$

From Lemma IV.1 it can be concluded that the bank angle limit of outermost wingman restricts the bank angle limit of the leader.

Lemma IV.2. If $W_{1}, W_{2}$ are two outer followers with $k_{1}, k_{2}$ such that $k_{1}>k_{2}>1$, then $\mathcal{R}_{W_{1}}^{V_{\max }} \subset \mathcal{R}_{W_{2}}^{V_{\max }}$. Where $\mathcal{R}_{W_{1}}^{V_{\max }}$ and $\mathcal{R}_{W_{2}}^{V_{\max }}$ are leader $V_{\max }$ saturation regions corresponding to $W_{1}$ and $W_{2}$, respectively.

Proof. Consider two outer UAVs $W_{1}$ and $W_{2}$ with formation parameters as $\rho_{c 1}, \theta_{c 1}$ and $\rho_{c 2}, \theta_{c 2}$, respectively. Let $\left(R_{l}^{*}, V_{l_{W_{1}}}^{*}\right)$ be the operating point of the leader where $W_{1}$ saturates in maximum airspeed and $\left(R_{l}^{*}, V_{l_{W_{2}}}^{*}\right)$ be the operating point where $W_{2}$ saturates in maximum airspeed. Using Eq. (31) for the two operating points,

$$
\begin{aligned}
& V_{l_{W_{1}}}^{*}=V_{\max } / k_{1} \\
& V_{l_{W_{2}}}^{*}=V_{\max } / k_{2}
\end{aligned}
$$

Since $k_{1}>k_{2}$, it implies

$$
V_{\max } / k_{1}<V_{\max } / k_{2}
$$

Using Eq. (42) and Eq. (43) in Eq. (44),

$$
\begin{aligned}
V_{l_{W_{1}}}^{*} & <V_{l_{W_{2}}}^{*} \\
\Rightarrow \mathcal{R}_{W_{1}}^{V_{\max }} & \subset \mathcal{R}_{W_{2}}^{V_{\max }}
\end{aligned}
$$

From Lemma IV.2 it can be concluded that the maximum airspeed of outermost wingman restricts the maximum airspeed of the leader.

Lemma IV.3. If $W_{1}, W_{2}$ are two inner followers with $k_{1}, k_{2}$ such that $k_{1} \leq k_{2} \leq 1$, then $\mathcal{R}_{W_{1}}^{V_{\min }} \subseteq \mathcal{R}_{W_{2}}^{V_{\min }}$. Where $\mathcal{R}_{W_{1}}^{V_{\text {min }}}$ and $\mathcal{R}_{W_{2}}^{V_{\min }}$ are $V_{\text {min }}$ saturation regions corresponding to $W_{1}$ and $W_{2}$, respectively.

Proof. Consider two outer UAVs $W_{1}$ and $W_{2}$ with formation parameters as $\rho_{c 1}, \theta_{c 1}$ and $\rho_{c 2}, \theta_{c 2}$, respectively. Let $\left(R_{l}^{*}, V_{l_{W_{1}}}^{*}\right)$ be the operating point of the leader where $W_{1}$ saturates in minimum airspeed and $\left(R_{l}^{*}, V_{l_{W_{2}}}^{*}\right)$ be the operating point where $W_{2}$ saturates in minimum airspeed. Using Eq. (31) for the two operating points,

$$
\begin{aligned}
& V_{l_{W_{1}}}^{*}=V_{\min } / k_{1} \\
& V_{l_{W_{2}}}^{*}=V_{m i n} / k_{2}
\end{aligned}
$$

Since $k_{1} \leq k_{2}$, it implies

$$
V_{\min } / k_{1} \geq V_{\min } / k_{2}
$$

Using Eq. (47) and Eq. (48) in Eq. (49),

$$
\begin{aligned}
V_{l_{W_{1}}}^{*} & \geq V_{l_{W_{2}}}^{*} \\
\Rightarrow \mathcal{R}_{W_{1}}^{V_{\min }} & \subseteq \mathcal{R}_{W_{2}}^{V_{\min }}
\end{aligned}
$$


Similarly, from the above lemma it can be concluded that the minimum airspeed of innermost wingman restricts the minimum airspeed of the leader.

Theorem IV.4. For multiple $U A V(n>2)$ formation, the operating region for the leader is the intersection of operating regions imposed by the innermost and the outermost UAV, respectively.

Proof. Consider $n$ vehicles in rigid formation, with one leader and $n-1$ followers. Let the number of outer UAVs and inner UAVs be $p$ and $q$, respectively with $p+q=n-1$. Let $W_{i}$, for $i=1,2, \ldots, p$ be the outer vehicles with $k_{i}$, for $i=1,2, \ldots, p$. The outer most vehicle $W_{\text {om }}$ has $k_{\text {om }}=\max \left\{k_{i}\right\}$, for $i=1,2, \ldots, p$. Therefore, from lemma IV.1

$$
\mathcal{R}_{W_{\text {om }}}^{\phi_{\max }} \subseteq \mathcal{R}_{W_{i}}^{\phi_{\max }}
$$

Similarly, from lemma IV.2

$$
\mathcal{R}_{W_{\text {om }}}^{V_{\max }} \subseteq \mathcal{R}_{W_{i}}^{V_{\max }}
$$

From Eq. (52) and Eq. (53), it can be stated that, the outer most UAV $W_{\text {om }}$ decides the limitation on bank angle $\phi_{l}^{\max }$ and the leader maximum speed $V_{l}^{\max }$. Similarly, the inner most vehicle $W_{i m}$ has $k_{i m}=\min \left\{k_{i}\right\}$, for $i=1,2, \ldots, q$. Therefore, from lemma IV.3

$$
\mathcal{R}_{W_{i m}}^{V_{\min }} \subseteq \mathcal{R}_{W_{i}}^{V_{\min }}
$$

Hence, the leader minimum speed limitations is limited by the innermost wingman. $V_{l}^{\text {min }}$ limitation on formation is decided by innermost UAV. The total operating region for multiple UAVs is the intersection of operating regions due to innermost and outermost UAVs

The total operating region in $\left(R_{l}, V_{l}\right)$ space is given by

$$
\mathcal{R}^{f} \equiv \mathcal{R}_{W_{\text {om }}}^{\phi_{\max }} \cap \mathcal{R}_{W_{\text {om }}}^{V_{\max }} \cap \mathcal{R}_{W_{\text {im }}}^{V_{\min }}
$$

The intersection region for a three UAV formation example with $V_{\max }=75 \mathrm{~m} / \mathrm{s}, V_{\min }=30 \mathrm{~m} / \mathrm{s}, \phi_{\max }=20^{\circ}$, $\rho_{c_{1}}=300 \mathrm{~m}, \theta_{c_{1}}=45^{\circ}, \rho_{c_{2}}=300 \mathrm{~m}$ and $\theta_{c_{2}}=-45^{\circ}$ is shown in figure 6 .

\section{Simulations}

A kinematic model of UAV with bank angle and airspeed saturations is used to verify the operating regions obtained in Section IV. Guidance law proposed in [11] is used for simulations. Maximum bank angle $\left(\phi_{\max }\right)$ is chosen as $\left|\phi_{\max }\right| \leq 20^{\circ}$ with maximum airspeed $V_{\max }$ as $75 \mathrm{~m} / \mathrm{s}$ and minimum airspeed as $30 \mathrm{~m} / \mathrm{s}$. From Eq. (7), the minimum radius of turn individual UAV at minimum speed corresponds to 252 $\mathrm{m}$. Three UAV rigid formation is considered for simulation with a leader and two followers. The formation parameters of followers are taken as $\rho_{c 1}=300 \sqrt{2} \mathrm{~m}, \theta_{c 1}=45^{\circ}$ and $\rho_{c 2}=300 \sqrt{2} \mathrm{~m}, \theta_{c 2}=-45^{\circ}$, respectively. From Eq. (22), the two followers $W_{1}$ (outer wingman) and $W_{2}$ (inner wingman), have $k_{1}>1$ and $k_{2}<1$, respectively. The leader operating region is generated using bank angle saturation region, $V_{\max }$ saturation region and $V_{\min }$ saturation region as shown in figure 7 . Four operating points are chosen, where operating point $\mathrm{A}$ is well within the operating region. Operating point $\mathrm{B}, \mathrm{C}$ and $\mathrm{D}$ violate $V_{\max }$ saturation region, $V_{\min }$ saturation region and bank angle saturation region respectively. The leader initial position is $(0,0)$ and the followers start at $(-300,-300)$ and $(300,-300)$, respectively. The leader and followers have an initial heading of $0^{\circ}$ and followers have initial airspeed of $50 \mathrm{~m} / \mathrm{s}$. Simulations are performed until first 100 seconds of flight. Results and observations for the simulations are presented in following subsections.

\section{A. Operating point A}

The leader operating point $\left(R_{l}, V_{l}\right) \mathrm{A}$ is $(1500 \mathrm{~m}, 50 \mathrm{~m} / \mathrm{s})$. Figure 8 shows the trajectories of leader and followers. Errors in $\rho$ and $\theta$ are plotted in figure 9 showing that the rigid formation is achieved in 35 seconds and is maintained thereafter. Figure 10, shows the airspeed and bank angles of leader and followers, respectively. It can be observed that spped and bank angle for all the vehicles are within flyable limits as analysed in this work. 


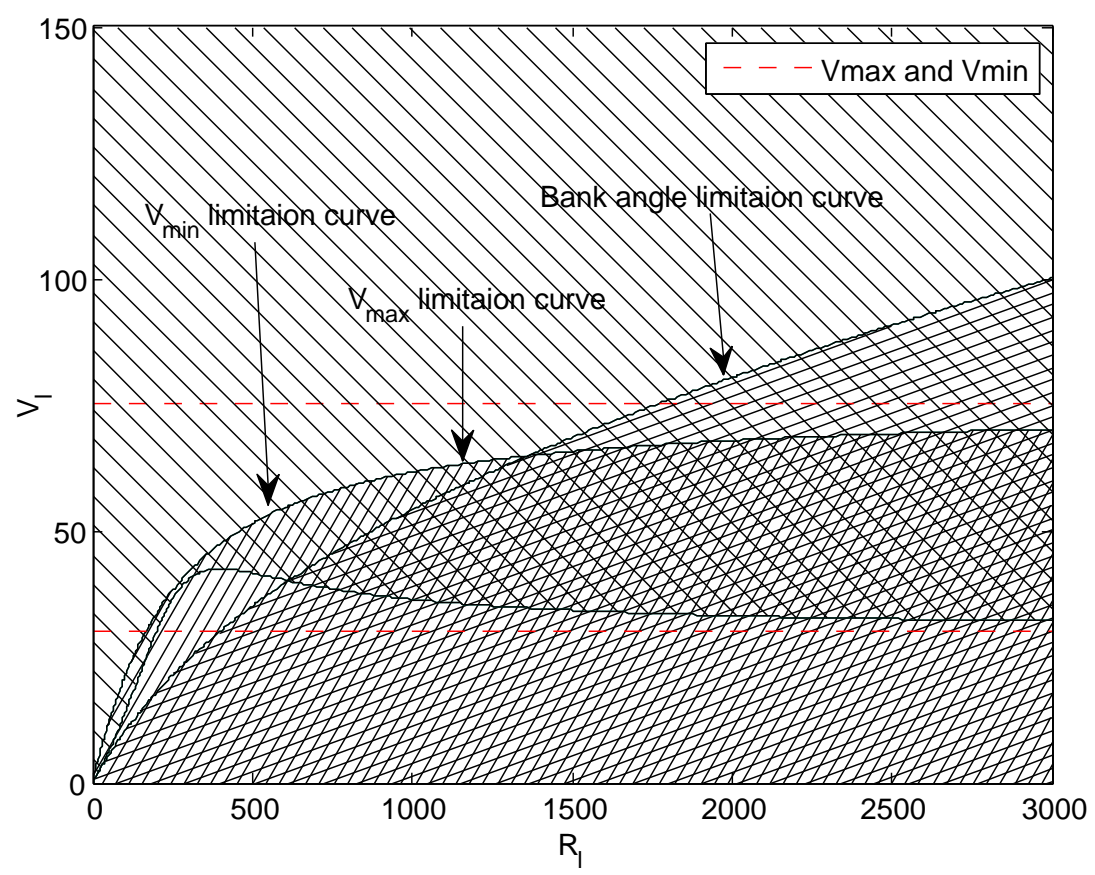

Figure 6. Operating region for multiple UAVs.

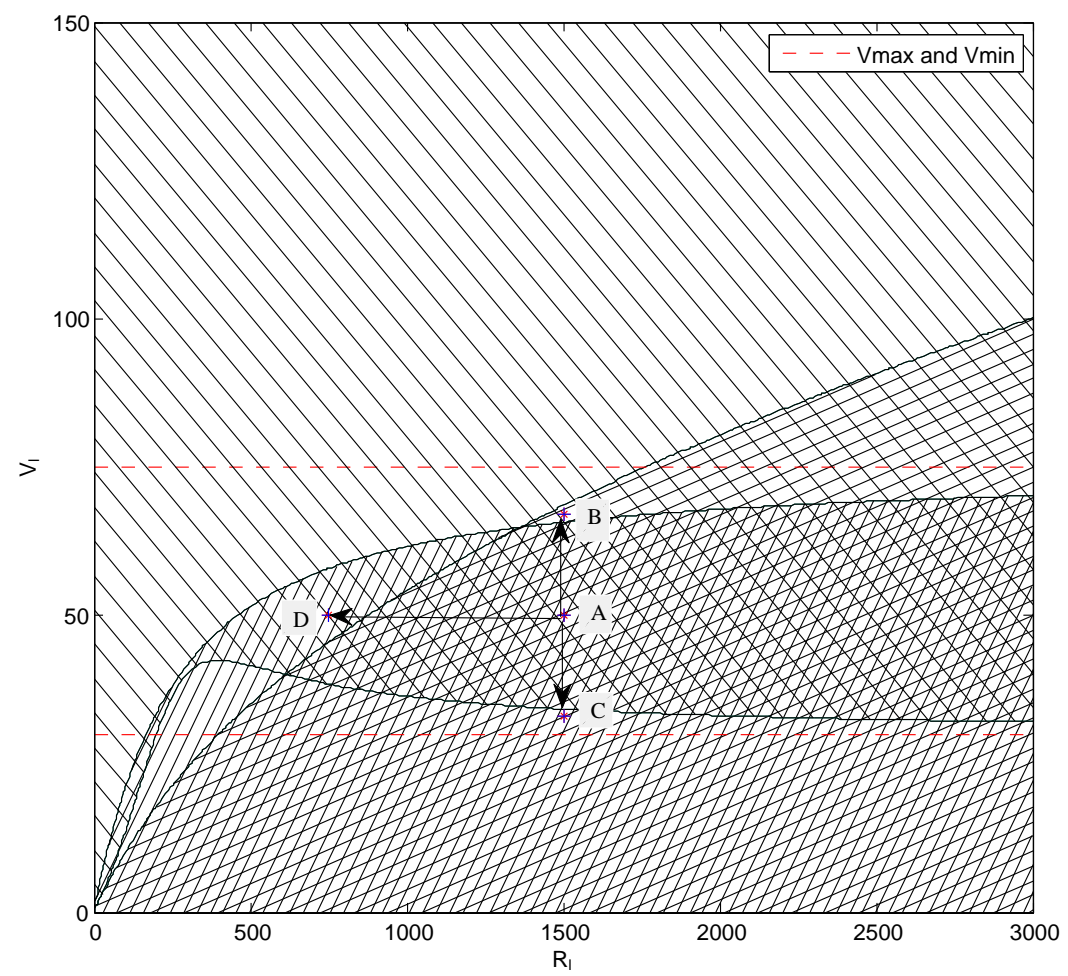

Figure 7. Operating points for simulations. 


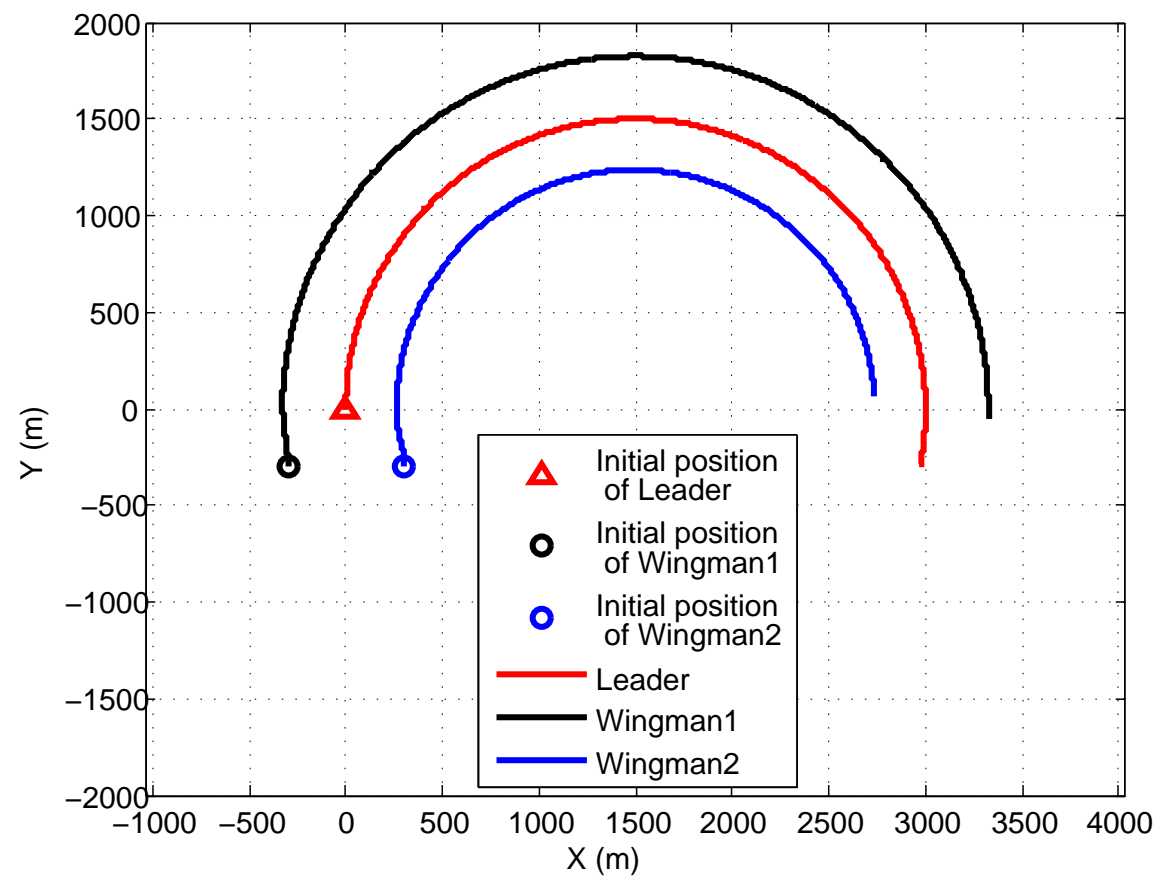

Figure 8. UAV trajectories for operating point A.
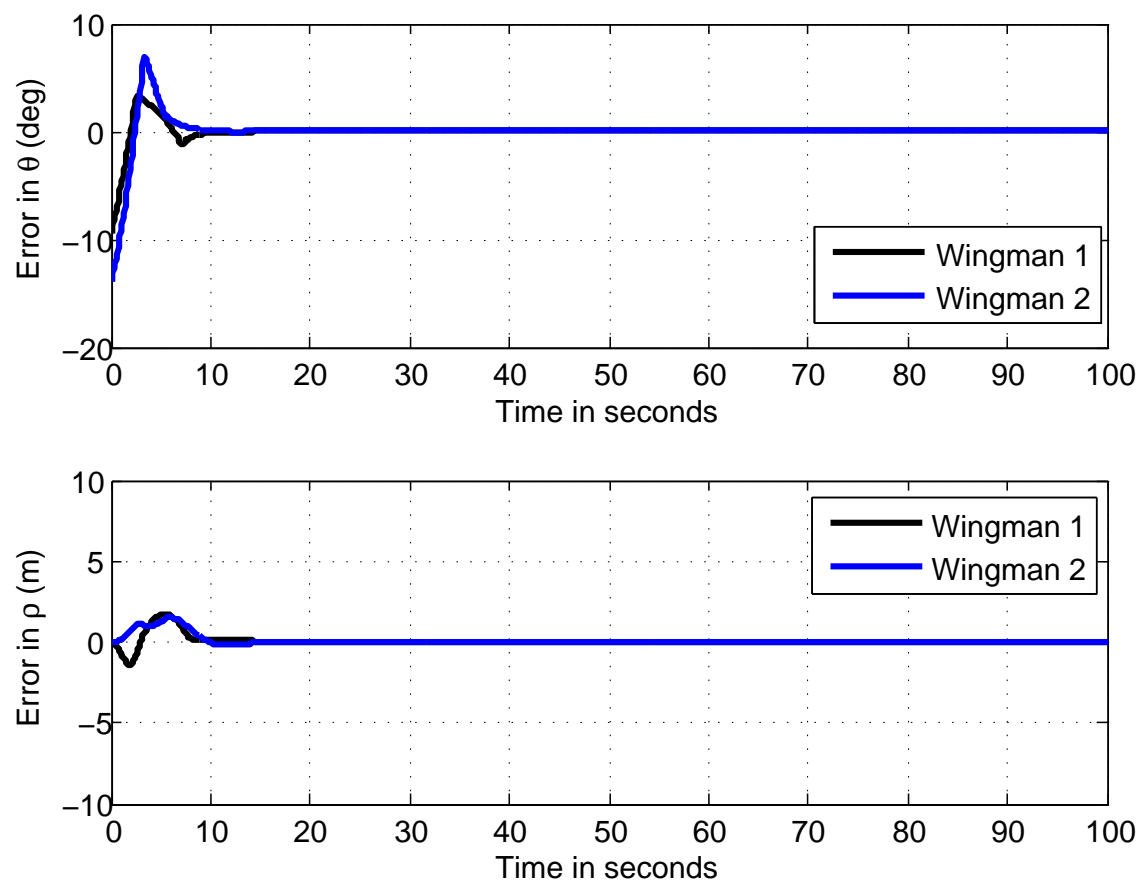

Figure 9. Errors for operating point A 

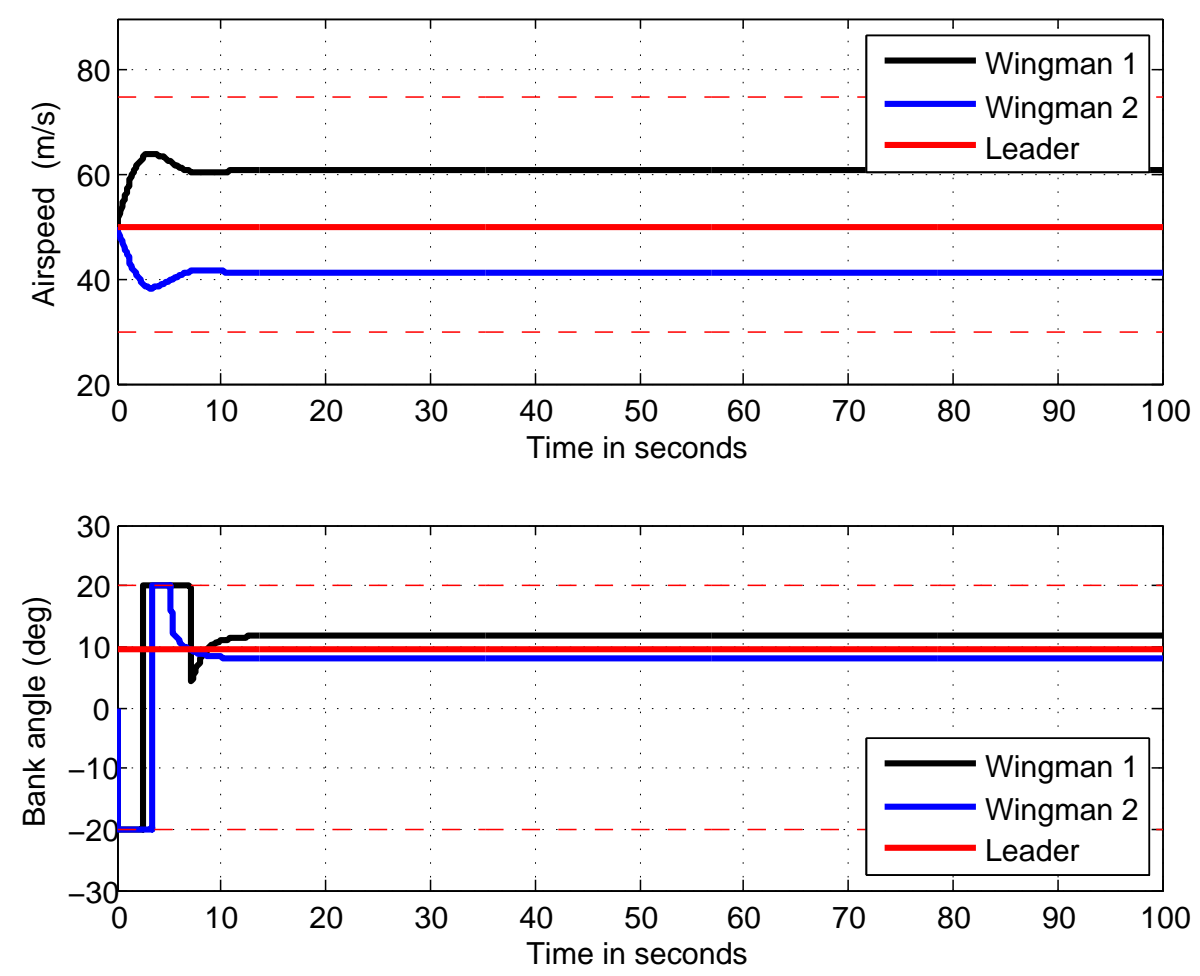

Figure 10. Velocities and headings for operating point A.

\section{B. Operating point transition from $\mathbf{A}$ to $\mathrm{B}$}

In this case, the operating point $\left(R_{l}, V_{l}\right)$ starts from point $\mathrm{A}(1500 \mathrm{~m}, 50 \mathrm{~m} / \mathrm{s})$ and after 40 seconds it gradually moves to point B $(1500 \mathrm{~m}, 67 \mathrm{~m} / \mathrm{s})$ which violates the $V_{\max }$ saturation constraint. Figure 11 shows the trajectories of leader and followers. Errors in $\rho$ and $\theta$ plotted in figure 12. Errors indicate that the outer wingman drifts out of the formation. Figure 13, shows the airspeed and bank angles for the vehicles. It can be observed that the outer UAV reaches saturation in maximum airspeed. This confirms to the analytic result of $V_{\max }$ saturation region given by Eq. (33).

\section{Operating point transition from $\mathrm{A}$ to $\mathrm{C}$}

In this case, the operating point moves from A to point $\mathrm{C}(1500 \mathrm{~m}, 33 \mathrm{~m} / \mathrm{s})$. Figure 14 shows the trajectories of leader and followers. Errors in $\rho$ and $\theta$ are plotted in figure 15. Errors show that the inner wingman drifts away from the formation. Figure 16, shows the airspeed and bank angles of the three vehicles. The inner wingman reaches stalling speed is unable to sustain a rigid formation. It can be observed that the inner UAV has reached saturation in minimum airspeed. The simulation validates the analysis of $V_{\min }$ saturation region given by Eq. (36).

\section{Operating point transition from $\mathbf{A}$ to $\mathbf{D}$}

In this case, the transition is from point A to D $(750 \mathrm{~m}, 50 \mathrm{~m} / \mathrm{s})$. Figure 17 shows the trajectories of leader and followers. Errors in $\rho$ and $\theta$ are plotted in figure 18 which shows that the outer UAV drifts from the formation. Figure 19, shows the airspeed and bank angles of leader and followers and it can be observed that the outer UAV saturates in bank angle. This is in accordance with analysis of bank angle saturation region given by Eq. (27). 


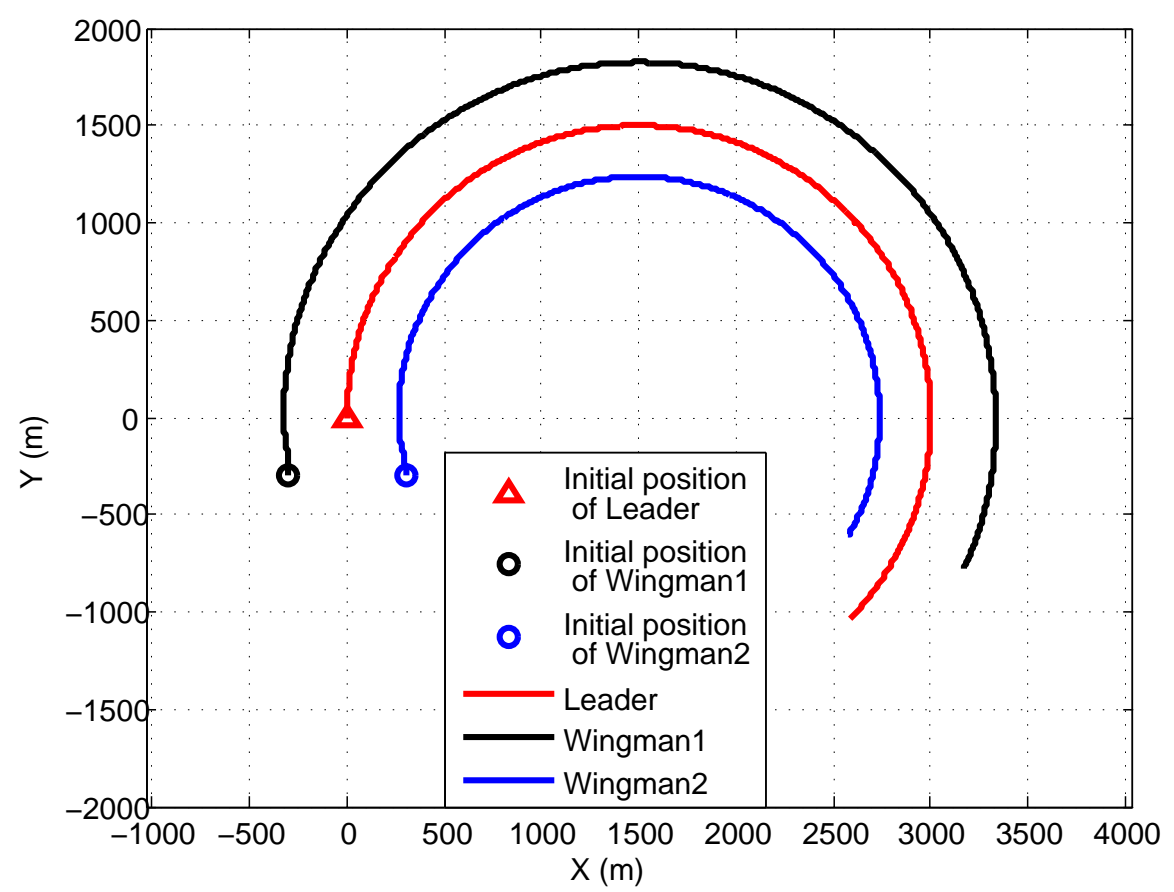

Figure 11. Trajectories for leader transition from operating point $\mathrm{A}$ to point $\mathrm{B}$.
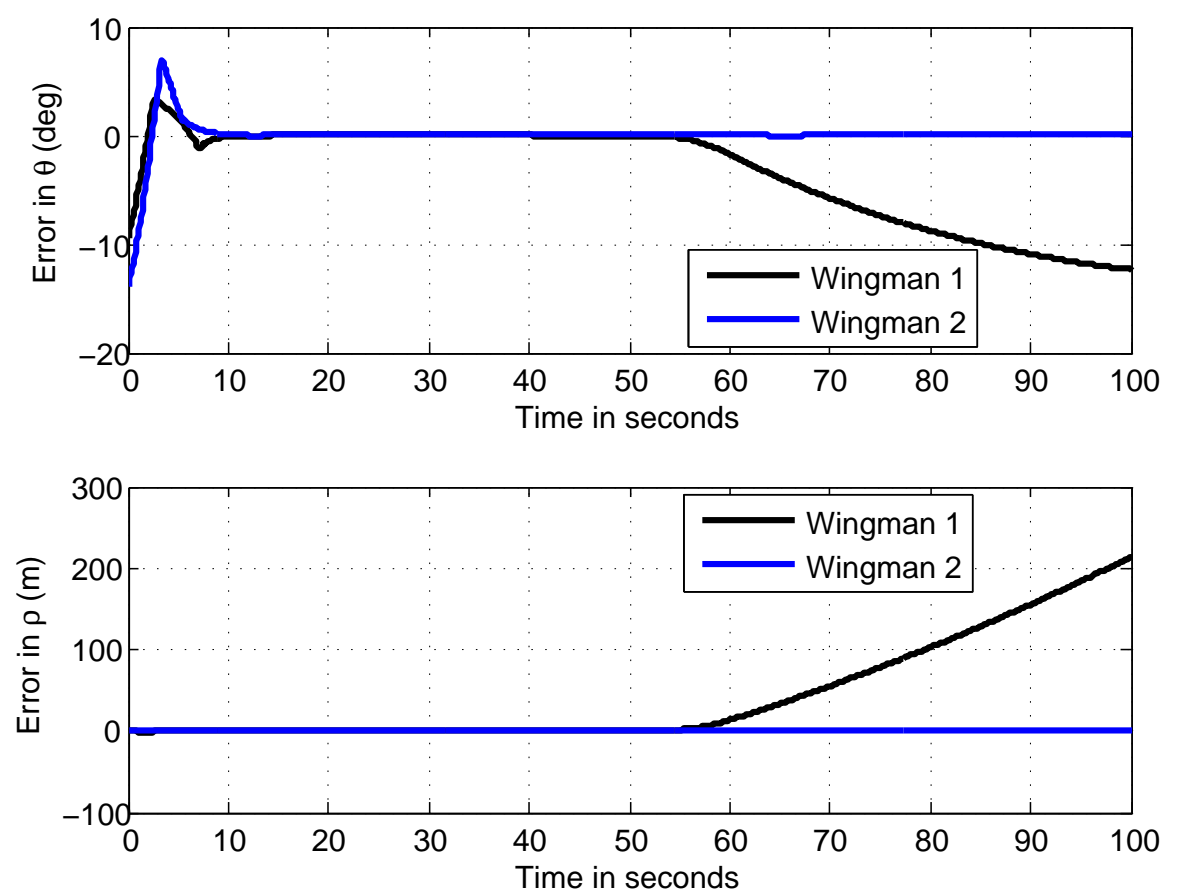

Figure 12. Errors for leader transition from operating point A to point B. 

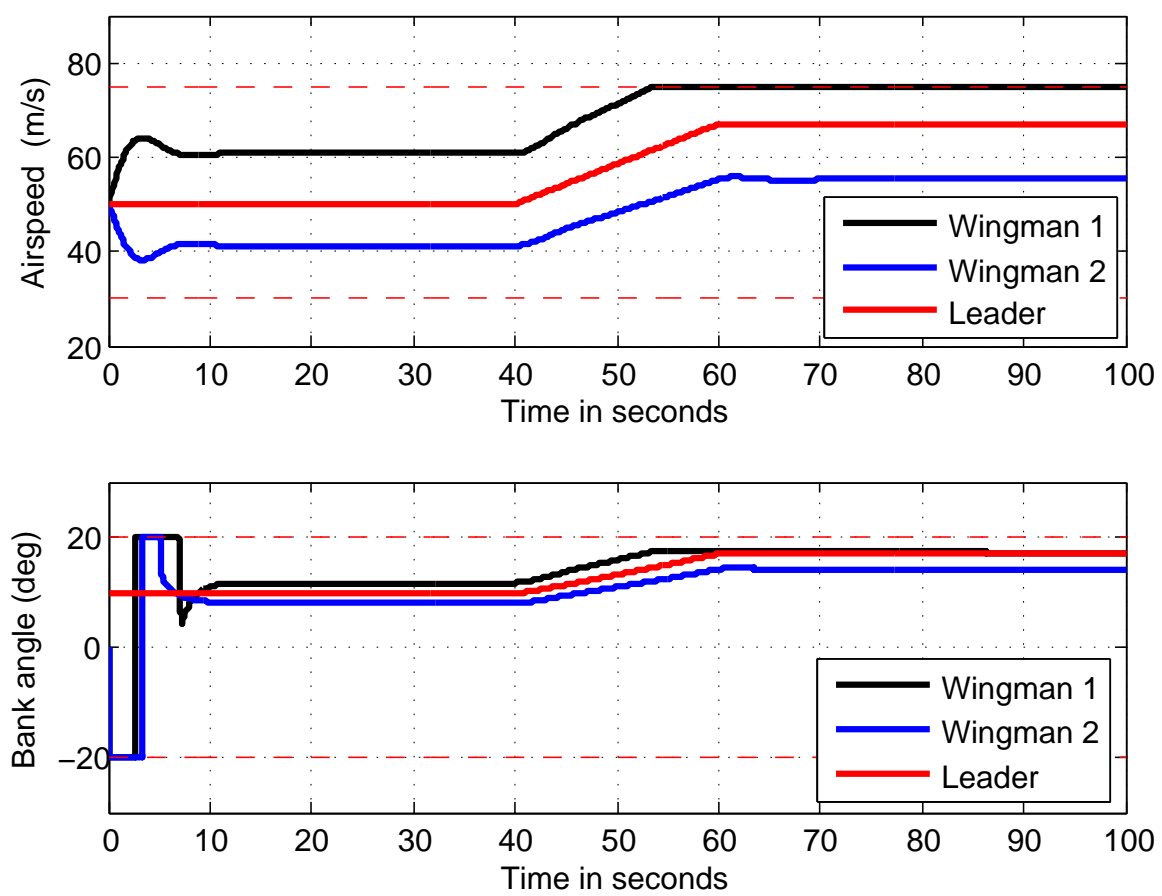

Figure 13. Velocities and headings for leader transition from operating point A to point $B$.

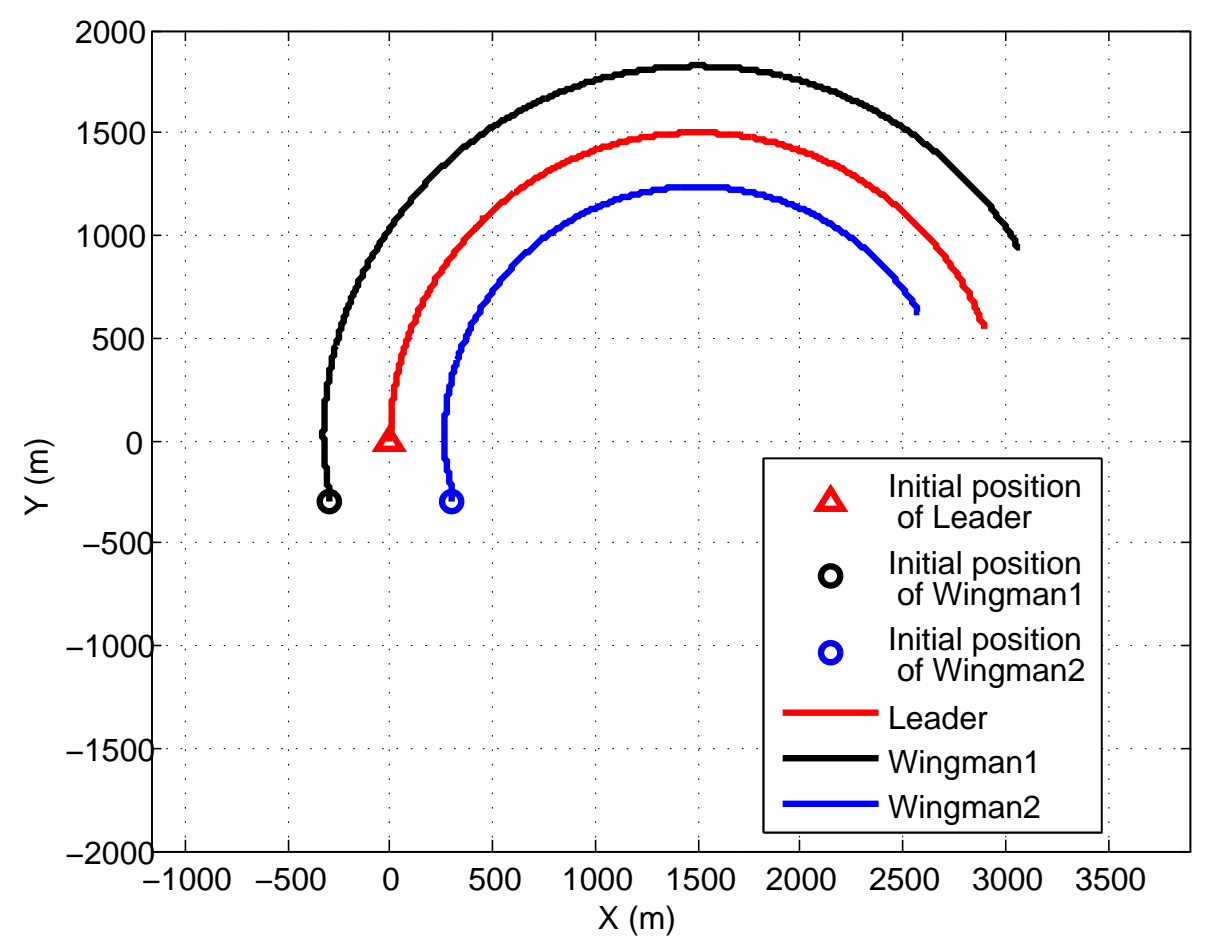

Figure 14. Trajectories for leader transition from operating point $\mathrm{A}$ to point $\mathrm{C}$. 

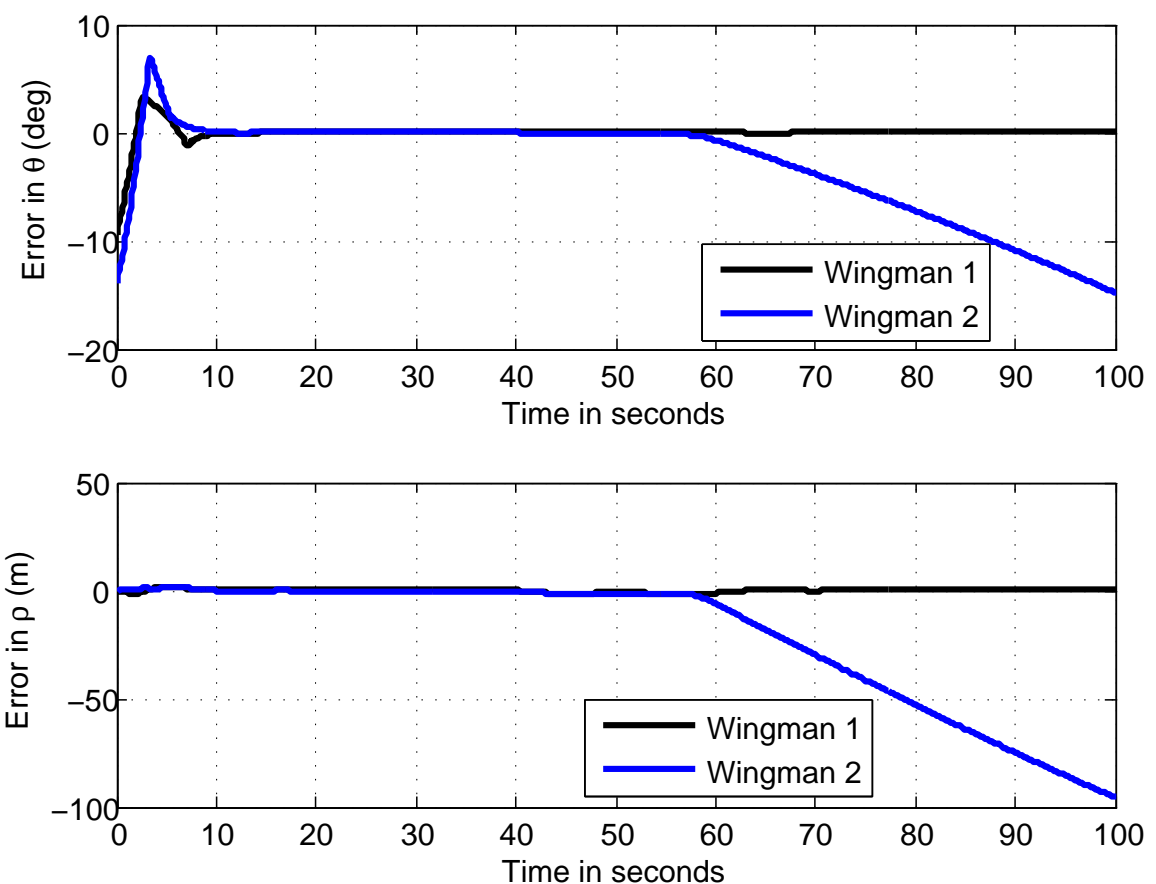

Figure 15. Errors for leader transition from operating point $\mathbf{A}$ to point $\mathbf{C}$.
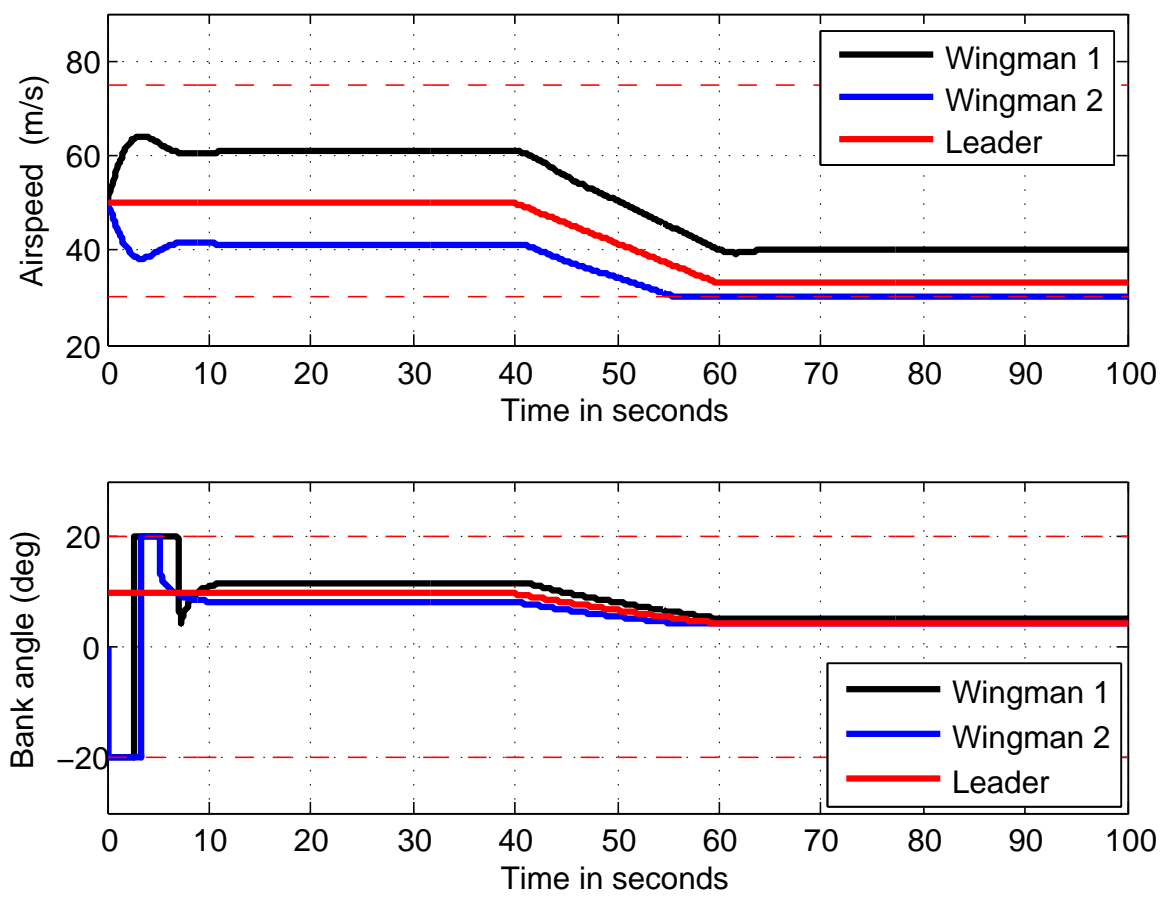

Figure 16. Velocities and headings for leader transition from operating point A to point C. 


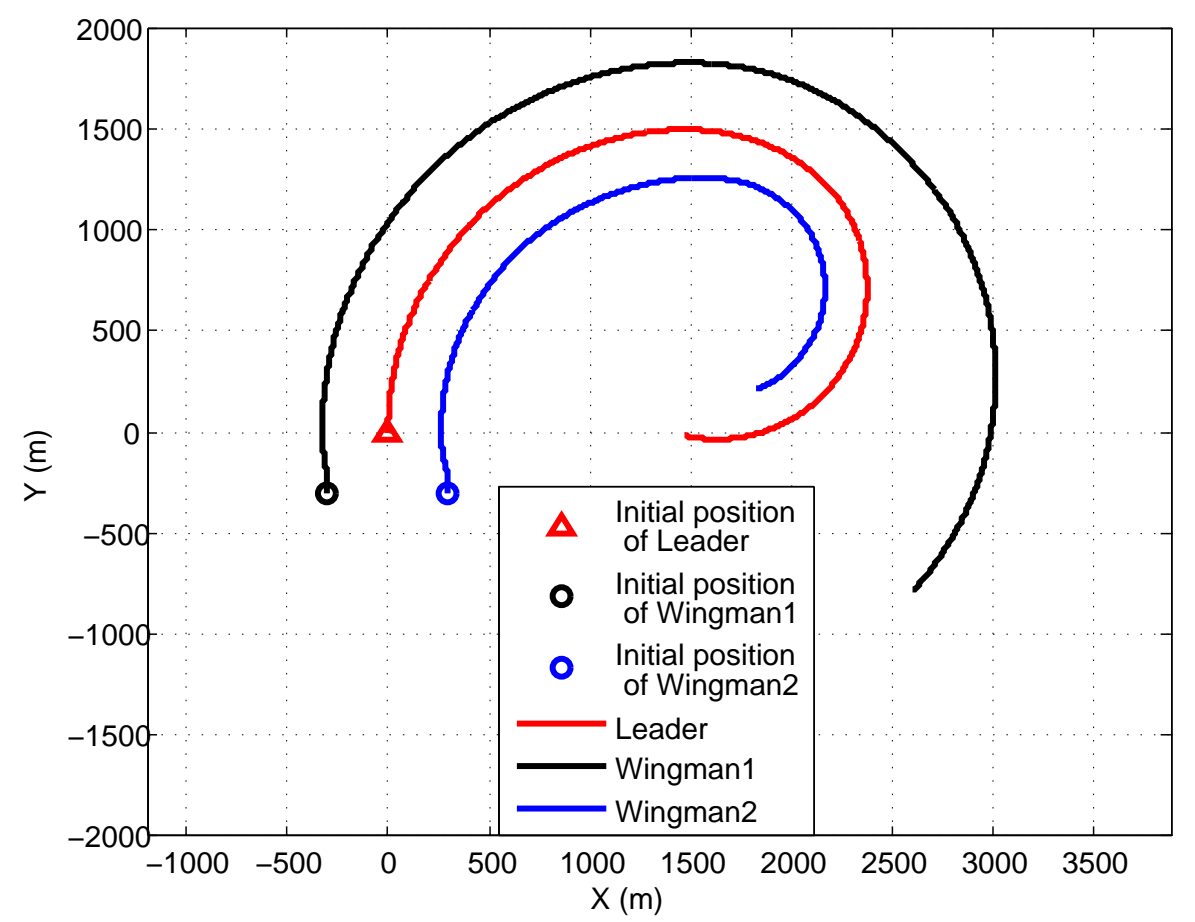

Figure 17. Trajectories for leader transition from operating point $A$ to point $D$.
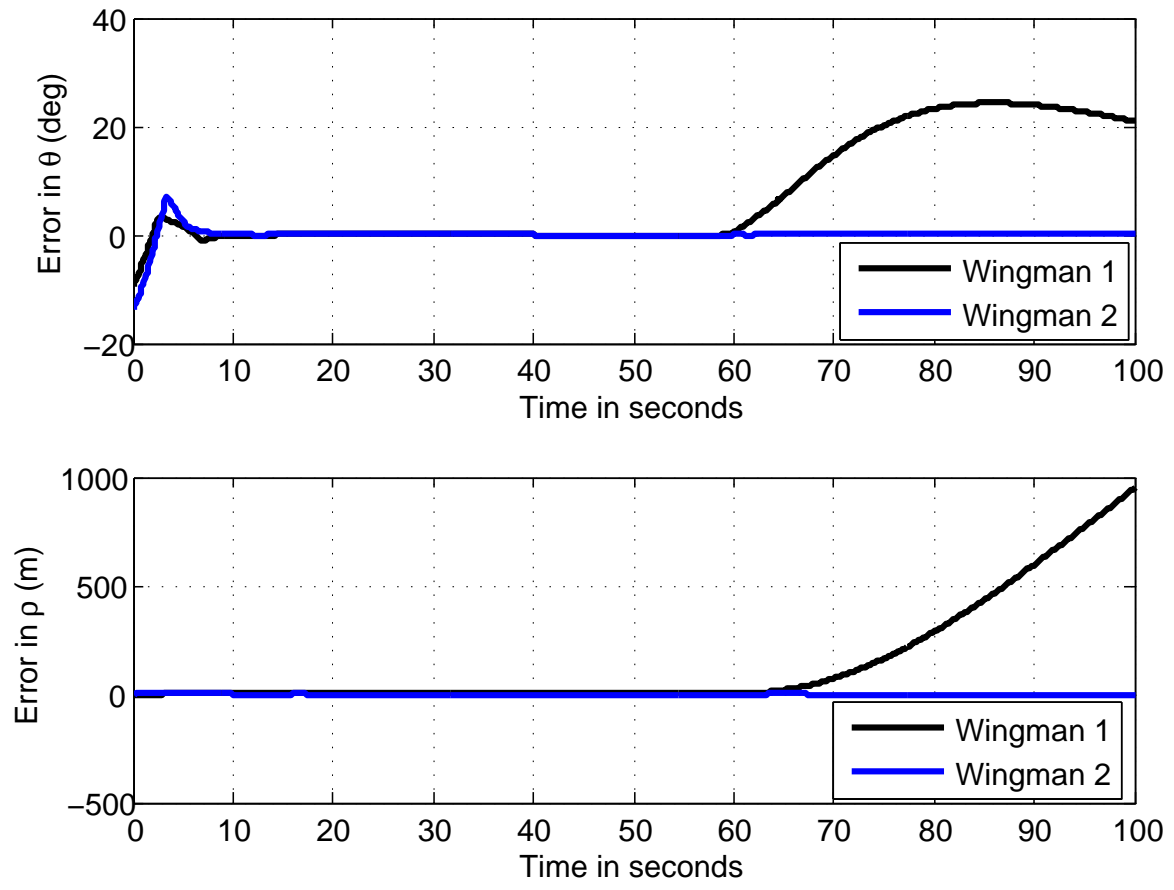

Figure 18. Errors for leader transition from operating point A to point D. 

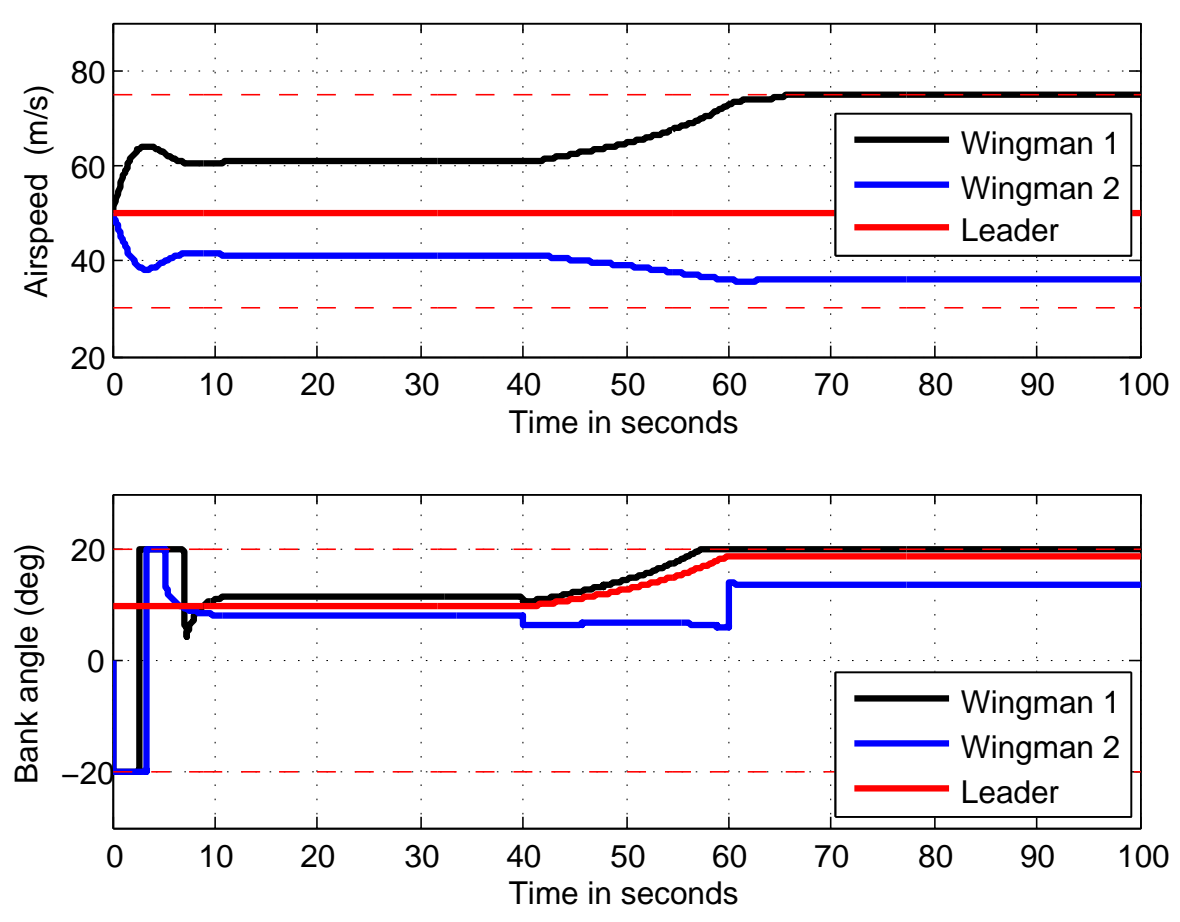

Figure 19. Velocities and headings for leader transition from operating point A to point D.

\section{Conclusions}

The aim of this work was to obtain an closed form operating region for leader subject to constraints by wingmen. Relation between kinematic constraints and formation kinematics were analysed. The analysis is used to define curves representing bounds on operating region for leader in $\left(R_{l}, V_{l}\right)$ space for a two UAV rigid formation. The bounds obtained are related to the kinematic constraints on the wingman. Scenarios with multiple wingmen were considered. And it was proven mathematically, that the leader operating region is effected only by the outermost and the innermost followers. Using this result the actual leader operating region was defined for formation with multiple followers. Simulations were performed for different operating points in the operating region of leader. Observations infer the compliance of simulation studies with the theoretical findings.

\section{References}

1 Tahk, M-J., Park, C-S., and Ryoo, C-K. "Line-of-Sight Guidance Laws for Formation Flight", Journal of Guidance, Control and Dynamics, Vol.28, No.2, 2005, pp. 708-716.

2 Pachter, M., D’Azzo, J.J., and Dargan. "Automatic Formation Flight Control", Journal of Guidance, Control and Guidance, Vol. 17, No. 6, 1994, pp. 1380-1383.

3 Fabrizio Giulietti, Lorenzo Pollini and Mario Innocenti. "Autonomous Formation Flight" IEEE Control System Magazine, Vol. 20, No. 6, 2000, pp. 34-44.

4 Jaydev P. Desai, James P.Ostrowski and R. Vijay Kumar "Modelling and Control of Formations of Nonholonomic Mobile Robots" IEEE Transactions on Robotics and Automation, Vol. 17, No. 6, 2001, pp. 905-908.

5 M. A. Lewis and K. H. Tan "High Precision Formation Control of Mobile Robotics using Virtual Structures" Autonomous Robots, Vol. 4, No. 4, 1997, pp. 387-403.

6 N. H. M. Li and H. H. T. Liu "Formation UAV Flight Control using Virtual Structure and Motion Synchronization" Proceedings of 2008 American Control Conference, 2008, pp. 1782-1787.

7 Chang Boon Low and Quee San Ng "A Flexible Virtual Structure Formation Keeping Control for Fixed-Wing UAVs" IEEE International Conferece on Control and Automation, 2011, pp. 621-626. 
8 Moscovitz, Y. and DeClaris, N. "Basic Concepts and Methods for Keeping Autonomous Ground Vehicle Formations", Proceedings of IEEE ISIC/CIRA/ISAS, pp. 14-17, September 1998.

9 Kar-Han Tan and M. Anthony Lewis "Virtual Structures for High Precision for Cooperative Mobile Robotic Control" 1996 IEEE/RSJ International Conference on Intelligent Robts and Systems.

${ }^{10}$ Chang Boon Low "A Dynamic Virtual Structure Formation Control for Fixed-Wing UAVs" IEEE International Conferece on Control and Automation, 2011, pp. 627-632.

${ }^{11}$ Challa, V.R. and Ratnoo, A. "Vector Field Based Formation Generation Guidance for Arbitrary Initial Conditions" Advances in Control and Optimization of Dynamical Systems, Vol. 3, No. 1, 2014, pp. 443-447.

12 Kwon, J-W. and Chwa, D. "Hierarchical Formation Control Based on a Vector Field Method for Wheeled Mobile Robots" IEEE Transaction on Robotics, Vol. 28, No. 6, 2012, pp. 1335-1345. 